\title{
Radiological diagnosis of hepatocellular carcinoma
}

This article was published in the following Dove Press journal:

Journal of Hepatocellular Carcinoma

9 September 2014

Number of times this article has been viewed

\section{Irene Bargellini \\ Valentina Battaglia \\ Elena Bozzi \\ Dario Luca Lauretti \\ Giulia Lorenzoni \\ Carlo Bartolozzi}

Department of Diagnostic and Interventional Radiology, Pisa

University Hospital, Pisa, Italy
Correspondence: Irene Bargellini Diagnostic and Interventional Radiology, University of Pisa,

Via Paradisa 2, 56100 Pisa, Italy

$\mathrm{Tel}+39050996961$

Fax +39050995 545

Email irenebargellini@hotmail.com
Abstract: Diagnosis of hepatocellular carcinoma (HCC) still remains a challenging issue. In the setting of liver cirrhosis, international guidelines have set the noninvasive criteria for HCC diagnosis, represented by the detection of contrast hyperenhancement in the arterial phase (wash-in) and hypoenhancement in the portal or delayed phase (wash-out) with dynamic multidetector computer tomography or magnetic resonance (MR) imaging. Although highly specific, this typical enhancement pattern has relatively low sensitivity, since approximately one-third of HCC nodules are characterized by atypical enhancement patterns. In atypical HCC nodules larger than $1 \mathrm{~cm}$, the majority of international guidelines recommend liver biopsy. However, there is an increasing interest in exploiting new noninvasive diagnostic tools, to increase the sensitivity of radiological diagnosis of HCC. Diffusion-weighted MR imaging and MR hepatobiliary contrast agents may represent useful tools for the detection and characterization of borderline hypovascular lesions by providing functional information such as water molecule motion in diffusion-weighted imaging and residual hepatobiliary function, which can be impaired early during the course of hepatocarcinogenesis. Also, dual-energy computed tomography (CT) represents an interesting new CT technology that could increase detectability and conspicuity of hypervascular lesions, thus possibly improving CT sensitivity in small HCCs. However, more data and further developments are needed to verify the usefulness of these new technologies in the diagnosis of HCC and to translate these recent advances into clinical practice.

Keywords: hepatocellular carcinoma, computed tomography, ultrasound, magnetic resonance imaging, liver cirrhosis, contrast agents

\section{Introduction}

Hepatocellular carcinoma (HCC) is the fifth most common cancer worldwide, the third cause of cancer-related death, and accounts for $7 \%$ of all cancers. ${ }^{1}$ The incidence of $\mathrm{HCC}$ is growing worldwide, and liver cirrhosis represents a major risk factor for the development of HCC. ${ }^{2,3}$ In Western countries, the most frequent underlying cause of cirrhosis is chronic hepatitis $\mathrm{C}$ virus infection, while in Africa and East Asia $60 \%$ of $\mathrm{HCC}$ is attributable to chronic hepatitis B virus infection; other causes of chronic liver injury are represented by alcohol abuse, aflatoxin exposure, cholestatic, autoimmune and metabolic disorders, and nonalcoholic steatohepatitis. ${ }^{4-6}$

The development of a neoplasm in cirrhosis is a long-lasting process. Many cellular changes occur along the pathway from normal hepatocytes to neoplastic cells, so different types of nodules can be detected in a cirrhotic liver, ranging from regenerative nodules to low-grade dysplastic nodules and high-grade dysplastic nodules, early HCC, and finally, overt HCC. ${ }^{7,8}$ 
Early diagnosis of HCC enables aggressive treatment, prolonging patients' long-term survival. Thus, international societies have developed ultrasound (US) surveillance programs for at-risk patients and specific imaging-based guidelines for definition of nodules over $1 \mathrm{~cm}$ in size detected during US surveillance. ${ }^{9-11}$

Proper definition of nodules as preneoplastic lesions or early HCC has critical implications. Dysplastic lesions should be followed by regular imaging studies, since at least one-third of them develop a malignant phenotype. ${ }^{12-14}$ On the other hand, early HCCs are treated with potentially curative procedures such as resection, transplantation, and percutaneous ablation. ${ }^{9}$

Until 2000, HCC diagnosis was based on biopsy. In 2001, a panel of experts first reported noninvasive criteria for HCC diagnosis based on a combination of imaging and laboratory findings. ${ }^{15}$ Since then, international guidelines have been updated, confirming the leading role of dynamic cross-sectional imaging as a noninvasive means to diagnose HCC in the setting of liver cirrhosis, thus limiting the diagnostic role of biopsy. ${ }^{8-11,14}$

\section{Hepatocarcinogenesis and imaging}

When a nodule becomes a dysplastic nodule, the normal hepatic arterial flow is decreased while portal venous flow is maintained. The evolution toward HCC is characterized by arterialization of the blood supply and sinusoidal capillarization. ${ }^{16}$

The presence of new unpaired arteries not accompanied by bile duct has been shown to be able to differentiate neoplastic nodules from regenerative nodules. ${ }^{17-19}$ The number of unpaired arteries gradually increases from cirrhotic nodules to HCC, and the degree of arterialization seems to correlate with HCC differentiation, particularly in lesions with a diameter less than $3 \mathrm{~cm}$. In fact, well-differentiated HCCs often receive preferential portal venous blood, whereas moderately and poorly differentiated tumors are supplied with arterial blood, as well as lesions beyond $3 \mathrm{~cm} .^{20}$

Sinusoidal capillarization is another common neoangiogenetic process. It involves transformation of fenestrated hepatic sinusoids into continuous capillaries, coupled with collagenization of the extravascular spaces of Disse and deposition of laminin and basement membranes near the endothelial cells and hepatocytes. ${ }^{21,22}$ As for arterialization of the blood supply, sinusoidal capillarization appears to be directly related to tumor differentiation. ${ }^{20}$

Together with these vascular changes, other modifications can be observed and exploited with current imaging techniques, such as a progressive decrease in the number of Kupffer cells and of bile canaliculi. ${ }^{23-28}$

The development of imaging techniques has led to a new paradigm in HCC diagnosis. In fact, as stated by the International Pathology Consensus Group for Hepatocellular Neoplasia in 2009, today noninvasive imaging can be able to define the different phases of hepatocarcinogenesis. ${ }^{8}$ As such, radiologists are playing a crucial role in staging $\mathrm{HCC}$ patients.

\section{HCC diagnosis: current guidelines}

The hemodynamic modifications occurring during hepatocarcinogenesis represent the pathological background for current noninvasive diagnosis of HCC in the setting of liver cirrhosis. This relies on postcontrast dynamic imaging techniques, particularly multidetector computed tomography (MDCT) and magnetic resonance (MR).

Dynamic MDCT and MR should be performed with state-of-the-art equipment enabling the acquisition of at least three different phases (arterial, portal venous, and equilibrium phases) after contrast injection and fixing the acquisition timing by proper techniques such as bolus chasing.

By dynamic imaging, HCC diagnosis is established by the detection of contrast hyperenhancement in the arterial phase (wash-in) and hypoenhancement in the portal venous and/or delayed phases of acquisition (wash-out) (Figure 1). This behavior is today defined as "HCC radiological hallmark". 9 In the setting of liver cirrhosis, this typical dynamic vascular pattern allows HCC diagnosis with almost $100 \%$ specificity and positive predictive value for nodules at least $1 \mathrm{~cm}$ in diameter. ${ }^{29-35}$

Although the HCC typical hallmarks at dynamic MDCT or MR imaging (MRI) are approved by all current guidelines, diagnostic algorithms differ in the suggested management of the detected nodules according to their size. ${ }^{36}$ The updated American Association for the Study of Liver Diseases (AASLD) guidelines suggest that the detection of the typical enhancement pattern at one single imaging modality in nodules larger than $1 \mathrm{~cm}$ suffices for diagnosing HCC. ${ }^{10}$ On the contrary, recent European Society for the Study of the Liver (EASL) guidelines underlined that data from prospective studies are still controversial regarding diagnostic accuracy of the typical radiological hallmarks for 1-2 cm lesions. Therefore, while a single imaging modality is considered sufficient for HCC diagnosis in nodules above $2 \mathrm{~cm}, 1-2 \mathrm{~cm}$ nodules should be investigated by two imaging modalities, if not performed in centers of excellence with "high-end radiological equipment". 9 Finally, Eastern guidelines disregard the dimensions of the nodules, stating that HCC diagnosis 

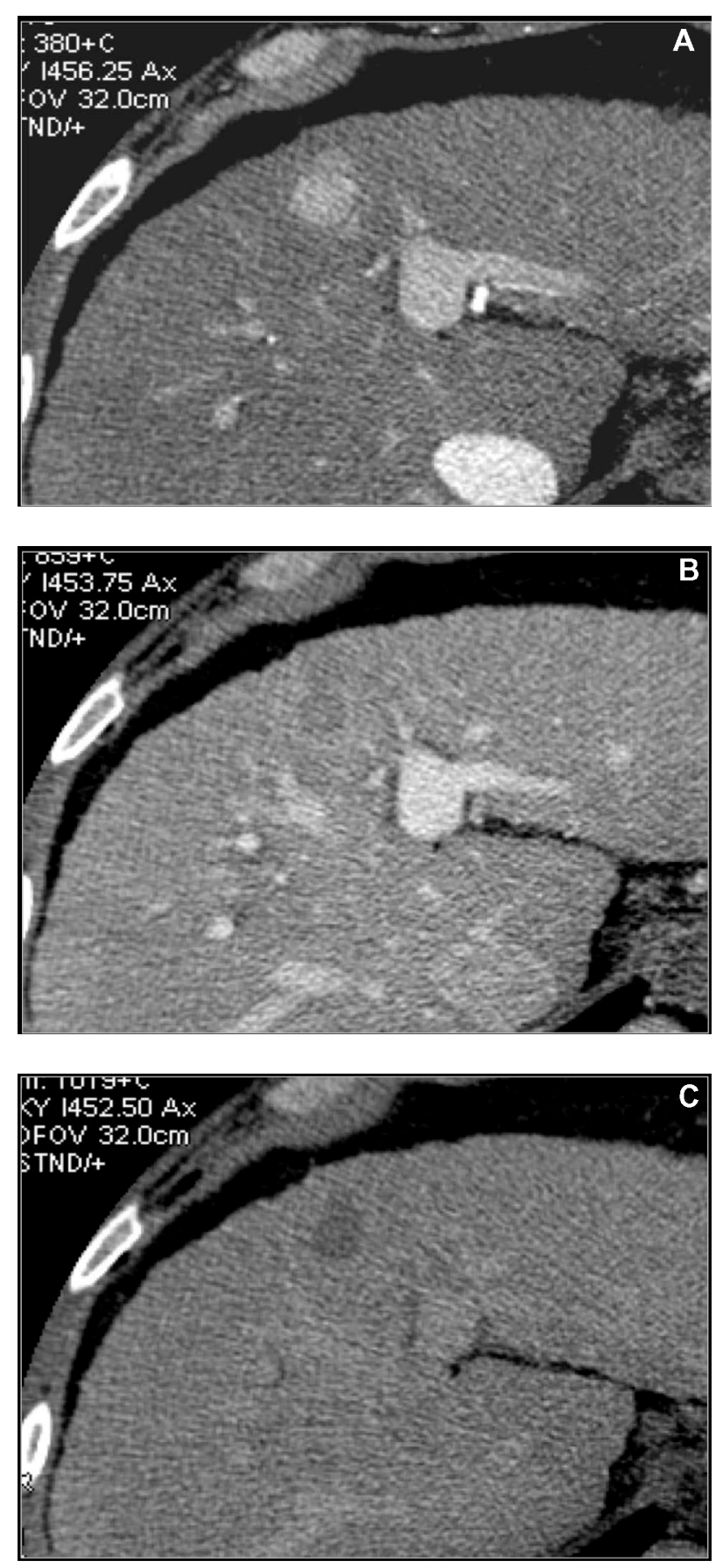

Figure I Hepatocellular carcinoma radiological hallmark.

Notes: Dynamic multidetector computed tomography demonstrates the presence of a nodule characterized by hyperenhancement in the arterial phase (wash-in) A) and hypoenhancement in the portal (B) and equilibrium (C) phases (wash-out) in the setting of liver cirrhosis. These features are currently the radiological hallmark for diagnosis of hepatocellular carcinoma.

can be established by the typical enhancement pattern even in lesions smaller than $1 \mathrm{~cm}^{11}$

\section{Imaging techniques}

While initial guidelines included MDCT, MR, and contrastenhanced US (CEUS) as equally valid dynamic imaging modalities for the diagnosis of $\mathrm{HCC},{ }^{37}$ later updates of AASLD and EASL guidelines have removed CEUS from this list. ${ }^{9,10}$

This exclusion has been justified by the report of few cases showing the typical HCC "wash-in/wash-out" pattern in histologically proven intrahepatic cholangiocarcinoma. ${ }^{30,38,39}$ This exclusion, however, has not been endorsed by all societies. For instance, the latest Italian Association for the Study of the Liver (AISF) position paper pointed out that the removal of CEUS from guidelines cannot at the moment be justified by the available evidence, considering the low incidence of intrahepatic cholangiocarcinoma, only half of them showing HCC radiological hallmarks, and the $>95 \%$ positive predictive value of CEUS. Nonetheless, AISF pointed out that since CT or MR is needed for tumor staging, the most cost-effective approach would be to prescribe first-line MR or CT, reserving CEUS to specific doubtful or inconclusive cases. ${ }^{40,41}$

Comparing MDCT with MR, international guidelines do not seem to privilege one imaging modality over the other. In fact, the vast majority of prospective studies report similar diagnostic accuracy. ${ }^{30}$ However, other studies reported higher sensitivity rates of MR compared with MDCT in the detection of the typical vascular pattern, particularly in 1-2 cm nodules (Figure 2). ${ }^{32,42}$ Moreover, MR offers some advantages in terms of identification and definition of cellular contents like iron, fat, glycogen, or blood. ${ }^{43}$ Finally, newly developed MR techniques such as diffusion-weighted imaging (DWI) and MR hepatobiliary (HB) contrast agents show promise for the characterization of atypical HCC nodules and for the assessment of the risk of degeneration of premalignant lesions.

\section{Advances in imaging techniques}

Only approximately two-thirds of HCC nodules are reported to show a typical vascular pattern, ${ }^{44}$ and diagnosis of atypical HCC nodules remains a controversial issue, with differences in their management in the available international guidelines. While both EASL and AASLD guidelines suggest biopsy for all atypical nodules larger than $1 \mathrm{~cm}, 9,10$ Eastern guidelines suggest the use of new diagnostic tools such as MR using reticuloendothelial system (RES), HB contrast agents or CEUS using Sonazoid, ${ }^{11}$ thus saving biopsy only for persistently doubtful or inconclusive findings.

Even the role of biopsy as a problem-solving tool appears controversial. In fact, it should be kept in mind that sampling errors can occur (such as insufficient tissue sampling or 

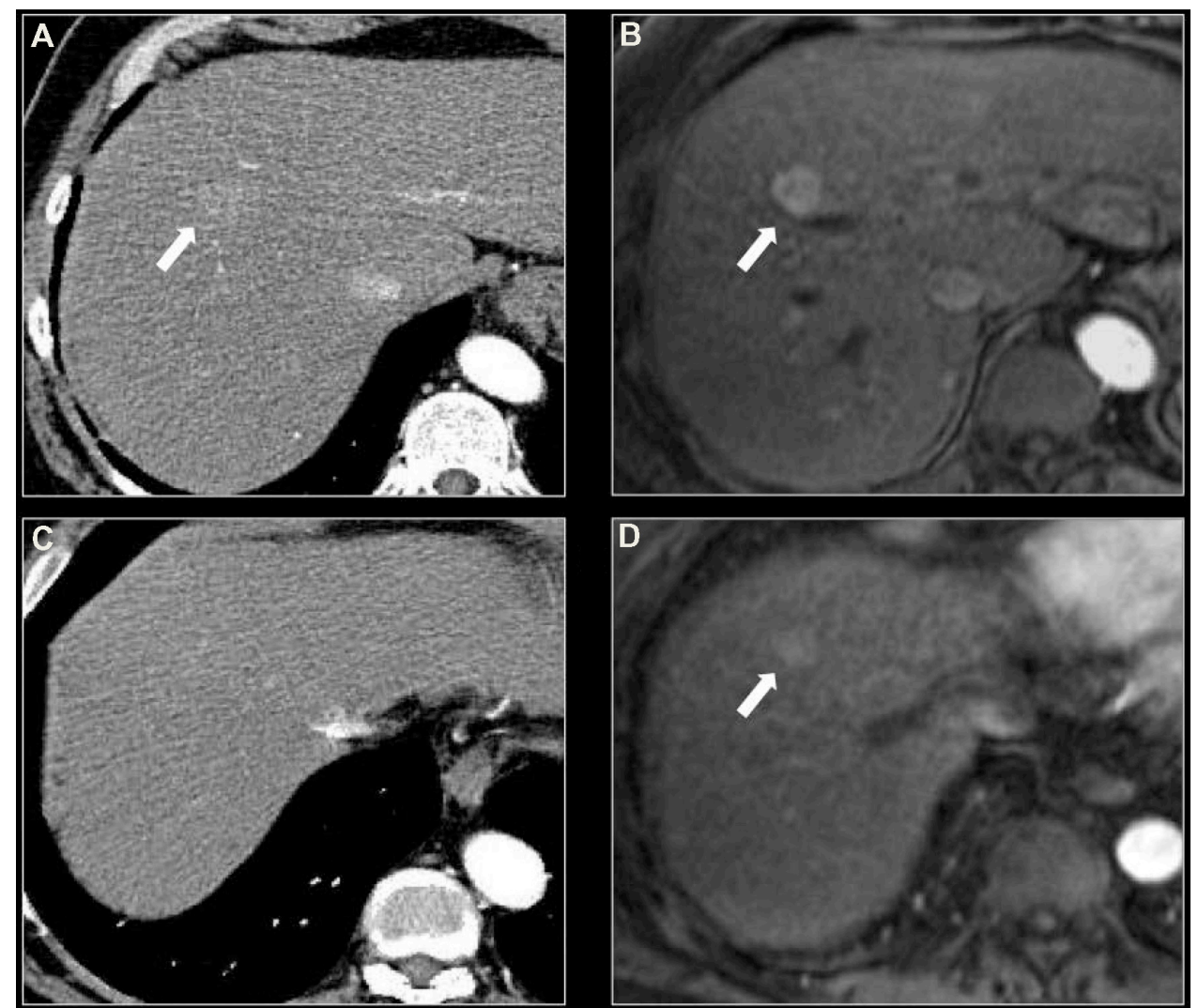

Figure 2 Multidetector computed tomography (MDCT) compared with magnetic resonance imaging (MRI).

Notes: Axial scans of the late arterial phase of MDCT (A and $\mathbf{C})$ and MRI (B and D). With MRI (B, arrow), the consipicuity is increased for the nodule that is only slightly visible with MDCT (A, arrow). Moreover, another hypervascular nodule is visible with MRI (D, arrow) that is not appreciated with MDCT.

samplings not representative of the entire lesions) and that pathological interpretation can be challenging on a specimen obtained from needle biopsy, with the inability to evaluate all the criteria suggesting malignancy (especially stromal invasion)..$^{29,33,45}$

The relatively low sensitivity of HCC radiological hallmarks and the issues regarding needle biopsy have raised attention to the possible role of newer diagnostic tools.

\section{DWI}

DWI is a dedicated MR sequence that allows the evaluation of the random motion (related to thermal effects) of water molecules ("Brownian motion") within biological tissues. Its application in the abdominal district, once limited, has become possible by the development of ultrafast sequences such as echoplanar imaging that enable the acquisition of an entire volume in a single breath-hold, thus reducing motion artifacts.

Recently, DWI has been introduced in liver MR protocols, as several studies have reported its usefulness in the detection and characterization of focal liver lesions, especially by quantifying the molecule motion through the apparent diffusion coefficient. ${ }^{46,47}$ Tumor areas will appear bright on high "b" values with DWI because of restriction of water molecules. ${ }^{48}$ Despite the increasing number of published papers focusing on DWI, ${ }^{49-51}$ no consistent data are yet available regarding its diagnostic accuracy in HCC. Therefore, DWI has not been incorporated in any international guidelines.

Nonetheless, DWI may be useful as an indicator of the risk of malignant transformation in borderline lesions. In fact, recent studies have reported that hypovascular nodules appearing as hyperintense in DWI have a higher risk of becoming hypervascular HCC nodules at subsequent follow-up (Figures 3 and 4). ${ }^{52}$

Thus, DWI may represent a valid adjunctive tool, providing additional information in borderline lesions, such as early HCC or high-grade dysplastic nodules.

\section{RES-targeted contrast agent}

RES-targeted contrast agents are superparamagnetic particles of iron oxide that determine alteration of the local magnetic field, resulting in signal loss in T2-weighted images. 

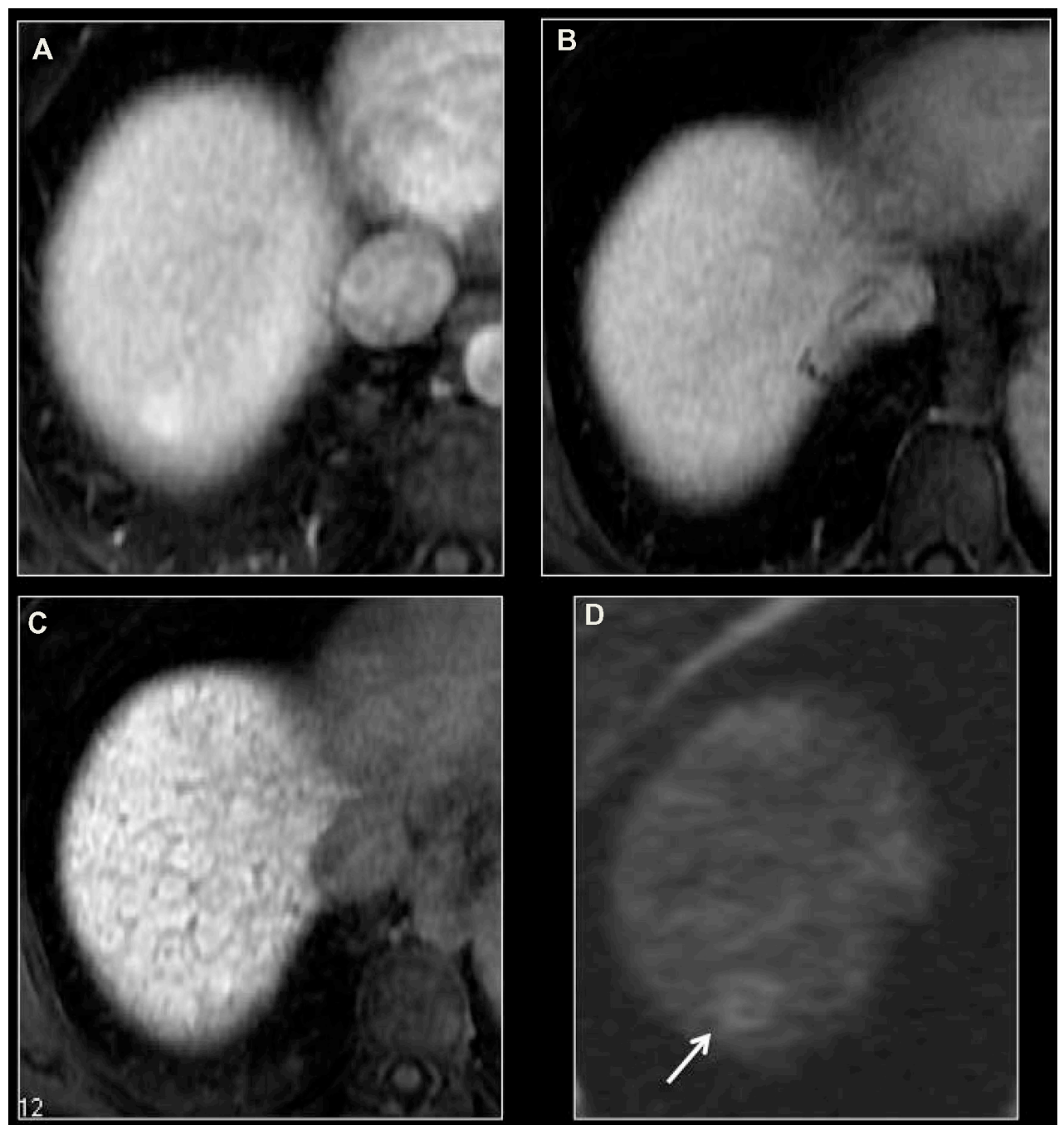

Figure 3 Dynamic imaging and diffusion-weighted magnetic resonance imaging.

Notes: With gadolinium ethoxybenzyl diethylenetriaminepentaacetic acid-enhanced magnetic resonance examination (A, arterial phase; $\mathbf{B}$, equilibrium phase; C, hepatobiliary phase), no areas of abnormal contrast enhancement are visualized. Diffusion-weighted imaging $(b=800)$ shows at the same level a nodular area of moderate hyperintensity (D, arrow).

Once injected, they are rapidly removed from the circulation by the RES, represented in the liver by the Kupffer cells, which remove more than $80 \%$ of the circulating contrast agent.

After contrast injection, it is possible to acquire first a standard dynamic vascular study, since small particles are spread within the blood, so that the influence on T2 relaxation time is limited. ${ }^{53}$ At each passage into sinusoids, superparamagnetic particles of iron oxide are taken up by the Kupffer cells, where they aggregate in clusters, inducing a strong shortening of $\mathrm{T} 2$ relaxation time, which can be appreciated approximately 10-20 minutes after injection.

Therefore, the signal loss of liver parenchyma is strictly related to the amount and metabolic activity of the Kupffer cells. Dysplastic nodules usually uptake the iron oxide particles and appear hypointense on T2-weighted images, although the number of Kupffer cells in these lesions may be variable, thus explaining the variable signal intensities. Accordingly, well-differentiated HCCs may still contain some Kupffer cells and could appear iso- or slightly hyperintense. ${ }^{54}$ Finally, moderately or poorly differentiated HCC will lose Kupffer cells and appear hyperintese. ${ }^{55}$

Even if RES contrast media can be used for the vascular dynamic study as substitutes of intravascular gadolinium (Gd) chelates, some studies have demonstrated overlap in enhancement patterns of variously graded HCCs and dysplastic nodules. In fact, the enhancement patterns of RES seem to differ from those obtained using Gd-based contrast agents, probably due to the relatively weak signal-to-noise ratio of RES on T1-weighted imaging. ${ }^{56}$ Therefore, it has been recommended to use RES only for the evaluation of the delayed T2 effect. 

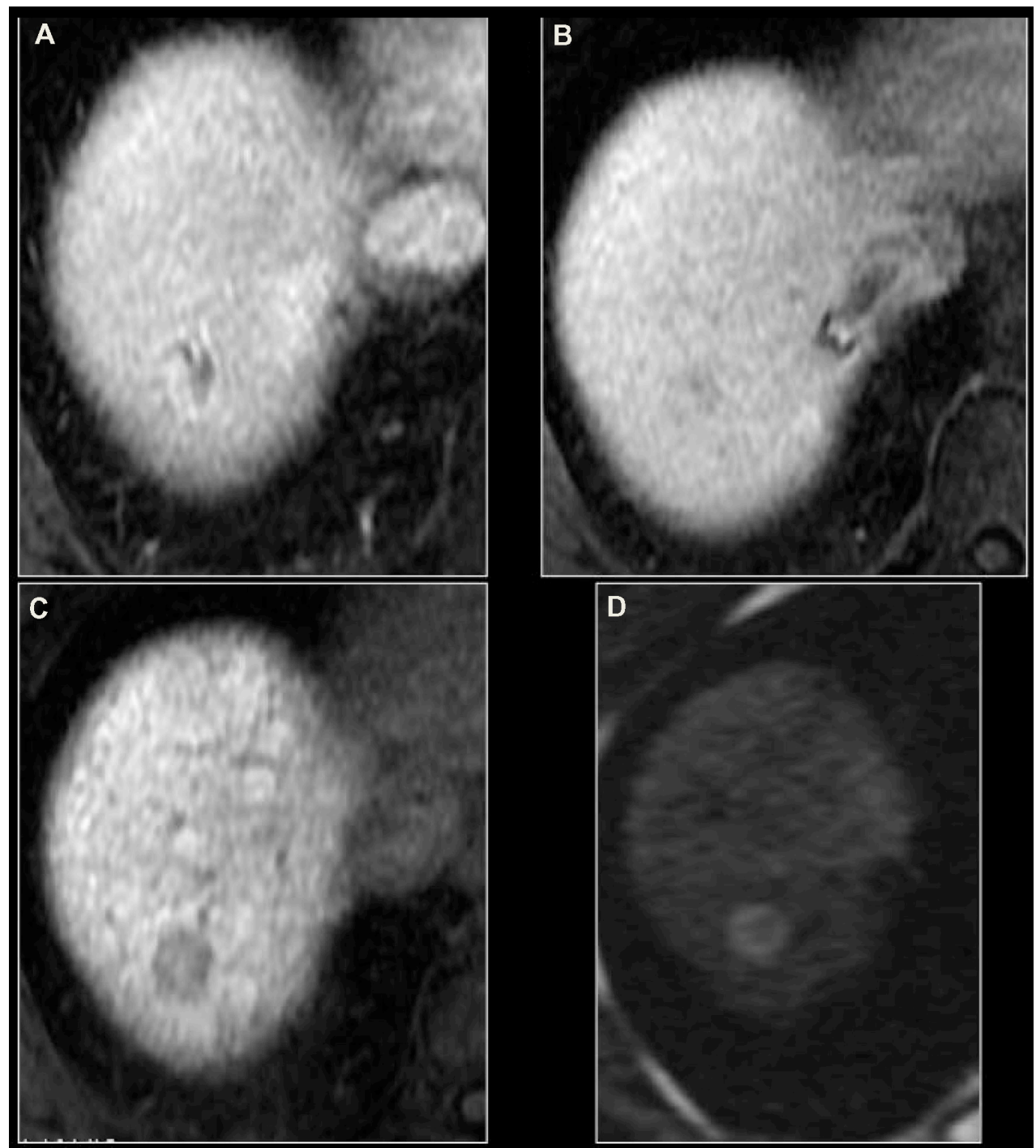

Figure 4 Same patient as in Figure 3 at 6-month follow-up.

Notes: At 6-month follow-up, gadolinium ethoxybenzyl diethylenetriaminepentaacetic acid-enhanced magnetic resonance examination (A, arterial phase; B, equilibrium phase; $\mathbf{C}$, hepatobiliary phase) demonstrates the increase in size of the nodule demonstrated with previous diffusion-weighted imaging, with in homogeneous yet typical enhancement pattern for hepatocellular carcinoma (A and B), hypointensity in the hepatobiliary phase (C), and hyperintensity with diffusion-weighted imaging (D, b=800).

The production of RES contrast agents was discontinued between 2008 and 2009 due to safety concerns.

\section{HB contrast agents}

HB contrast agents are paramagnetic Gd chelates that determine the shortening of T1 relaxation time. Thanks to their lipophilic characteristics, these agents, after the intravascular/interstitial distribution, are taken up by functioning hepatocytes, metabolized, and excreted into the bile. ${ }^{57}$ The contrast uptake and excretion are predominantly related to the molecular transporter organic anion-transporting polypeptide 8 (OATP8) and multidrug resistance-associated protein 2 (MRP2). ${ }^{58-60}$ The current contrast agents used in clinical practice are gadobenate dimeglumine (Gd-BOPTA/Dimeg, MultiHance; Bracco, Milan, Italy) and gadolinium ethoxybenzyl diethylenetriaminepentaacetic acid (Gd-EOB-DTPA, Primovist; Bayer Schering Pharma, Germany). The main difference between these two agents is the percentage of the injected dose that can be excreted in the biliary tract (approximately 50\% of administered Gd-EOBDTPA and $5 \%$ of Gd-BOPTA). ${ }^{61}$

OATP8 appears to be downregulated during malignant transformation of hepatic nodules. ${ }^{58}$ Therefore, hypointensity in the HB phase (HBP) is suggestive of premalignancy or malignancy, with almost all HCCs and some highgrade dysplastic nodules being hypointense in this phase (Figure 5). ${ }^{44}$

It has been demonstrated that $\mathrm{HB}$ contrast agents are able to increase the sensitivity for the diagnosis of $\mathrm{HCC}$, especially 


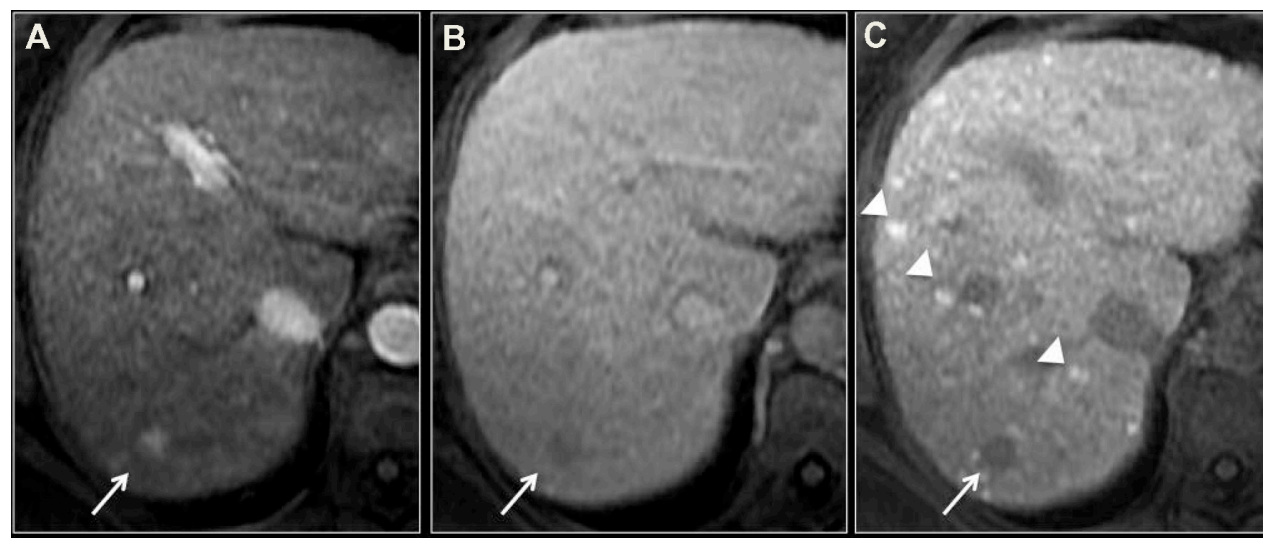

Figure 5 Signal intensity in the hepatobiliary phase.

Notes: Gadolinium ethoxybenzyl diethylenetriaminepentaacetic acid-enhanced magnetic resonance examination (A, arterial phase; B, equilibrium phase; C, hepatobiliary phase) demonstrates the presence of a nodule characterized by late arterial phase wash-in and delayed phase wash-out, typical for hepatocellular carcinoma (arrows). This nodule appears hypointense in the hepatobiliary phase, as confirmation of malignancy. In the hepatobiliary phase, multiple hyperintense nodules are also displayed (arrow heads) that do not show enhancement in the dynamic acquisition, as for dysplastic nodules.

for lesions smaller than $2 \mathrm{~cm}^{42,62-66}$ and for borderline lesions such as hypovascular HCC. ${ }^{67}$

The nodular contrast enhancement seems to decrease in line with the degree of nodule differentiation. ${ }^{67}$ In fact, in well-differentiated HCC, neoplastic cells are still able to uptake the contrast medium and metabolize it while there is a loss of excretion activity; thus, the nodules may appear as isointense or even hyperintense in the HBP. On the contrary, in poorly differentiated HCC, the cells have completely lost the normal metabolic functions, and the nodules will appear hypointense in the HBP because of the lack of contrast agent uptake. Of interest, these modifications can be considered an early event of hepatocarcinogenesis, preceding portal blood flow reduction and neoangiogenesis. ${ }^{68}$

Unfortunately, the HB enhancement does not allow differentiating between DN and hypovascular well-differentiated HCCs, and this represents the major limitation of HB contrast agents, not allowing at the moment the inclusion of these agents in international guidelines.

Moreover, approximately $10 \%$ of overt HCCs also show iso or high signal intensity in the HBP. This phenomenon can be explained by a genetic alteration that results in the overexpression of OATP8 and MRP2. ${ }^{58,69,70}$

The increased sensitivity of HB-enhanced MRI may impact the clinical management particularly of early-stage HCC patients. A recent paper has demonstrated that in Barcelona Clinic for Liver Cancer (BCLC) stage 0 and A patients, the information added by Gd-EOB-DTPA-enhanced MRI to MDCT can modify treatment decisions in up to onethird of patients; in particular, $12 \%$ of the included patients were found to be beyond Milan criteria for transplantation by MRI compared with MDCT, due to the increased number of detected HCC nodules. ${ }^{71}$

Finally, the combination of HB contrast agents, enhanced MRI, and DWI can result in a significantly higher accuracy and per-lesion sensitivity for detecting small $(\leq 2 \mathrm{~cm}) \mathrm{HCCs}$ compared with either imaging set alone. ${ }^{72}$

\section{Dual-energy CT}

A recent development in $\mathrm{CT}$ has been the introduction of dual-source technology, ${ }^{73}$ consisting of two X-ray tubes that can be operated at different tube currents. Dual-energy CT (DECT) implies simultaneous acquisition of datasets at two different photon spectra in a single CT examination, ${ }^{74}$ resulting in the ability to reconstruct the data at $80 \mathrm{kVp}$, $140 \mathrm{kVp}$, and the weighted average. The use of the two tubes at different tube currents enables the differentiation of materials of nonequal radiographic density.

Recent papers have reported on the use of DECT in the diagnosis of $\mathrm{HCC}$, demonstrating that low $\mathrm{kVp}$ images may increase sensitivity in detecting hyperattenuating lesions after contrast injection, such as hypervascular HCC. ${ }^{75}$

Because there is increased photoelectric absorption at $80 \mathrm{kVp}$ relative to the absorption at $140 \mathrm{kVp}$, contrast material has higher attenuation at lower peak voltage. This increases the lesion-to-liver ratios, enables depiction of small lesions, or, alternatively, enables a reduction in the contrast material injected.

However, this increase in sensitivity goes along with a decrease in the image quality for low $\mathrm{kVp}$ images. This might not represent a clinical problem in hypervascular lesions, being compensated by the high lesion-to-liver ratio. $^{75}$ 
More recently introduced spectral CT equipment consists of a single-detector and a single-source DECT system with the capability for fast switching between two peak voltage settings ( $140 \mathrm{kVp}$ and $80 \mathrm{kVp}$ ). Using this technology it is possible to reconstruct conventional polychromatic images corresponding to $140 \mathrm{kVp}$ and monochromatic images with energies ranging from $40 \mathrm{keV}$ to $140 \mathrm{keV}$. This new technology can reduce beam hardening artifacts and optimize contrast with selectable monochromatic energy $(\mathrm{keV})$. As a result of the reduced number of photons in the X-ray spectrum at the lower energy portion, image noise is expected to increase when photon energy decreases. In this setting, an adaptive iterative reconstruction algorithm has been incorporated to reduce image noise.

Iodine-based contrast material provides greater X-ray attenuation at lower tube voltages, and hyperenhancing hepatic lesions become more conspicuous at lower energies. ${ }^{76}$ It has been demonstrated that $\mathrm{HCC}$ detectability and conspicuity increases at $40-70 \mathrm{keV}$ monochromatic imaging by improving contrast-to-noise values (Figure 6). ${ }^{76}$

Moreover, spectral CT provides iodine-based material decomposition images that appear to be highly sensitive in the identification of spots of contrast uptake and therefore in the detection of small HCC nodules (Figure 6). By material decomposition it is also possible to quantify iodine concentration in the lesions, obtaining a quantitative parameter that reflects the blood supply of lesions, which could be useful in the differential diagnosis of HCC and in the follow-up after treatment. ${ }^{77-80}$

\section{Toward standardization of interpretation and reporting}

Currently, findings at liver imaging are inconsistently interpreted and reported by most radiologists. Thus, scoring systems have been developed in an attempt to reduce interobserver variability in liver imaging interpretation and reporting.

The first system was proposed by the US Organ Procurement and Transplant Network (OPTN)/United Network for Organ Sharing (UNOS) in 2008 in an attempt to reduce false-positive imaging diagnoses, set minimum requirements for imaging protocols, and standardize reporting of lesions. ${ }^{81}$ This classification system emphasizes specific, well-defined imaging features of HCC, with stringent criteria for lesions between $1 \mathrm{~cm}$ and $2 \mathrm{~cm}$, in order to maximize diagnostic specificity ${ }^{82}$ and ensure proper organ allocation. However, sensitivity seems to be relatively low using OPTN criteria, particularly for smaller lesions.$^{83}$ Moreover, precise
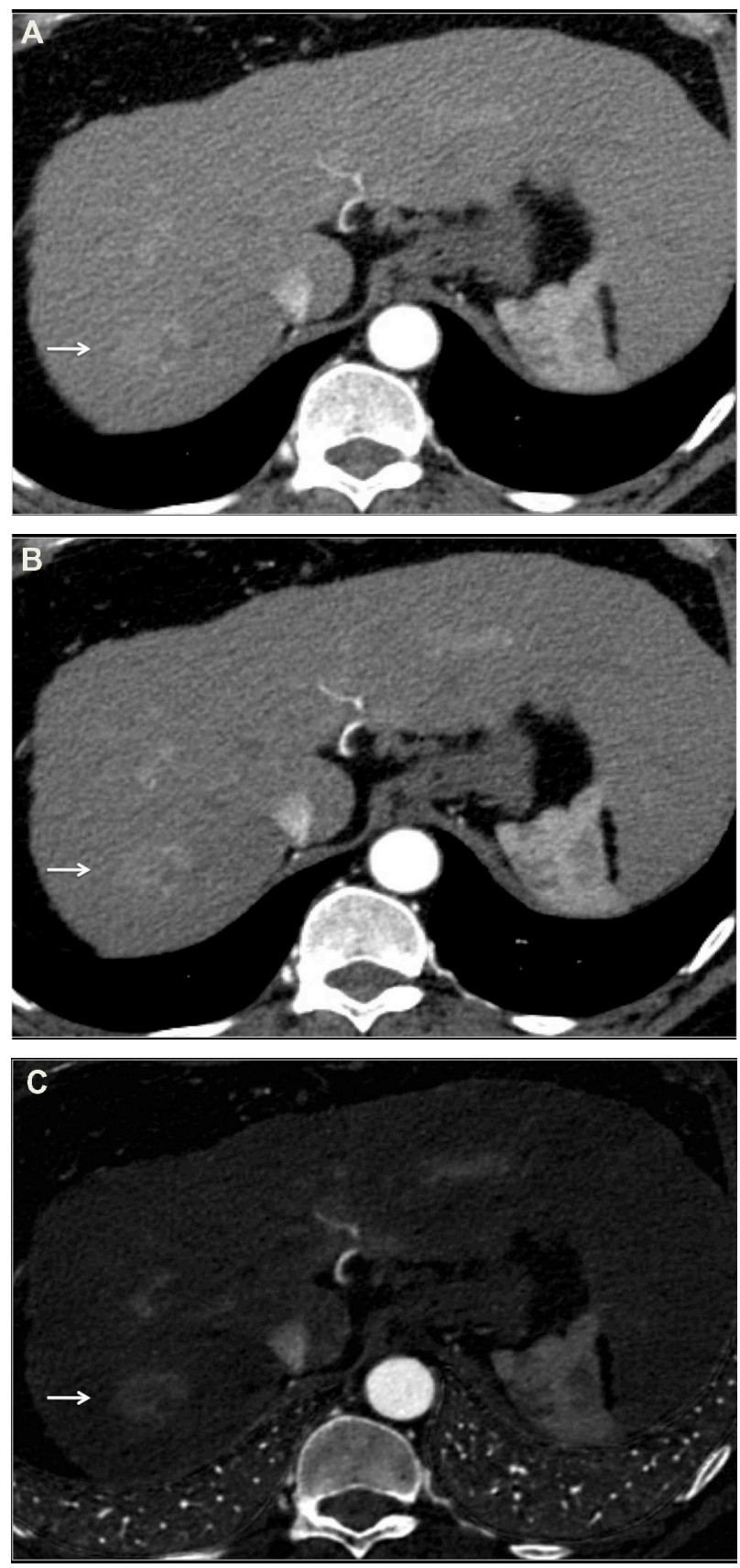

Figure 6 Dual-energy and spectral imaging computed tomography.

Notes: Axial scan in the late arterial phase of dual-energy computed tomography examination. The $140 \mathrm{kV}$ p polichromatic image shows an ill-defined, slightly hyperattenuating area (A, arrow). This area is better depicted in the $70 \mathrm{keV}$ monochromatic image (B, arrow), while its conspicuity is increased in the iodinebased material decomposition image (C, arrow). The latter is characterized by lower image quality, which, however, is compensated by the evident increase in lesion-to-liver contrast ratio.

definitions and illustrative examples are lacking, introducing ambiguity in image interpretation.

In 2012, the American College of Radiology developed the Liver Imaging-Reporting and Data System (LI-RADS), which includes five categories (from definitely benign to definitely HCC) on the basis of several criteria, such as 
Table I Sensitivity and specificity of different imaging modalities for the diagnosis of HCC

\begin{tabular}{|c|c|c|c|c|}
\hline Author & No of pts and no of nodules & Imaging modality & Sensitivity (\%) & Specificity (\%) \\
\hline \multicolumn{5}{|l|}{ Dynamic imaging } \\
\hline \multirow[t]{2}{*}{ Forner et $\mathrm{al}^{29}$} & 89 pts, 89 nodules $<2 \mathrm{~cm}$ & CEUS & 51.7 & 93.1 \\
\hline & & D-MRI & 61.7 & 96.6 \\
\hline \multirow[t]{3}{*}{ Sangiovanni et $\mathrm{al}^{30}$} & 64 pts, 55 HCC I-2 cm & CEUS & 26 & 100 \\
\hline & & $\mathrm{D}-\mathrm{CT}$ & 44 & 100 \\
\hline & & D-MRI & 44 & 100 \\
\hline \multicolumn{5}{|l|}{ DWI } \\
\hline \multirow[t]{2}{*}{ Vandecaveye et $\mathrm{al}^{49}$} & 55 pts, II 4 lesions & DWI-MRI & 95.2 & 82.7 \\
\hline & & D-MRI & 80.6 & 65.4 \\
\hline \multirow[t]{2}{*}{$X u$ et $a^{50}$} & 54 pts, 59 nodules & DWI-MRI & 67.5 & 94.7 \\
\hline & & D-MRI & 82.5 & 57.9 \\
\hline \multirow[t]{3}{*}{ Wu et $\mathrm{al}^{51}$} & Pooled estimated analysis & DWI & $73-82$ & $84-86$ \\
\hline & & D-MRI & 79 & 62 \\
\hline & & $D W I+D-M R I$ & 93 & 84 \\
\hline \multicolumn{5}{|l|}{ MRI HB contrast agents } \\
\hline \multirow[t]{2}{*}{ Golfieri et $\mathrm{al}^{42}$} & 127 pts, 62 atypical nodules & $U+D-M R I$ & 88.4 & 88 \\
\hline & & $U$ + D-MRI + HB-MRI & 99.4 & 95 \\
\hline \multirow[t]{2}{*}{ Ahn et $\mathrm{al}^{62}$} & 59 pts, 84 nodules & $U+D-M R I$ & $83.3-85.7$ & $86.9-91.7$ \\
\hline & & $U$ + D-MRI + HB-MRI & $82.8-93.1$ & $89.7-93.1$ \\
\hline \multirow[t]{3}{*}{ Haradome et al ${ }^{64}$} & 52 pts, $60 \mathrm{HCC}$ & $\mathrm{D}-\mathrm{CT}$ & $68.3-7 \mid .7$ & 94.9 \\
\hline & & D-MRI & $75-78.3$ & $92.3-94.9$ \\
\hline & & D-MR + HB-MRI & 86.7 & 89.7-92.3 \\
\hline \multirow[t]{4}{*}{ Di Martino et $a^{166}$} & 140 pts, $163 \mathrm{HCC}$ & CEUS & 71 & 62 \\
\hline & & $\mathrm{D}-\mathrm{CT}$ & 71 & 87 \\
\hline & & D-MRI & 71 & 87 \\
\hline & & D-MRI + HB-MRI & 87 & 90 \\
\hline
\end{tabular}

Abbreviations: CEUS, contrast-enhanced ultrasound; D-CT, dynamic computed tomography; D-MRI, dynamic magnetic resonance imaging; DWI-MRI, diffusion-weighted imaging MRI; HB, hepatobiliary; HCC, hepatocellular carcinoma; MRI, magnetic resonance imaging; pts, patients; U + D-MRI, unenhanced and dynamic MRI.

mass-like configuration, arterial phase hyperenhancement, portal venous phase or later phase hypoenhancement, increase in size, presence of tumor capsule, and vein involvement. ${ }^{84}$ Moreover, LI-RADS defines minimum acceptable technical parameters for CT and liver MRI and proposes a lexicon for controlled imaging terminology in an attempt to standardize reports' content and structure, improve communication with clinicians, and facilitate decision making, outcome monitoring, auditing, and research.

In the latest 2013.1 version, LI-RADS and UNOS OPTN categorizations have been unified. As a result of these changes, LR 5 and OPTN 5 categories are now congruent, and UNOS defers OPTN $1-4$ categories to LR $1-4 .{ }^{85}$ This version applies to multiphase CT and MRI examinations performed with conventional extracellular contrast materials. Work is ongoing to include MRI with HB agents and CEUS. ${ }^{86}$

Although the repeatability of LI-RADS diagnostic criteria and scores is still questioned, ${ }^{82}$ the implementation of this or similar scoring systems could improve image interpretation and patients' management and open the way to the development of semiautomated structured reports, ultimately increasing radiologist efficiency and communication capabilities. ${ }^{87}$

\section{Conclusion}

Diagnosis of HCC still remains a challenging issue. Since early diagnosis has a strong impact on chances for curative treatments and long-term prognosis, US surveillance for at-risk populations is of outmost importance.

In the setting of liver cirrhosis, noninvasive diagnosis of HCC is made possible by the very high specificity and positive predictive value of the so-called "HCC radiological hallmarks", represented by the detection of arterial phase wash-in and portal or delayed phase wash-out at dynamic MDCT or MR examinations.

Although highly specific, these hallmarks lack sensitivity, since approximately one-third of HCC nodules in cirrhotic liver do not show this typical enhancement pattern. This is more frequent in lesions smaller than $2 \mathrm{~cm}$ and in well-differentiated HCC. While the majority of international guidelines suggest the use of liver biopsy to set the diagnosis of atypical HCC, there is an increasing interest in exploiting new noninvasive diagnostic tools to increase sensitivity for HCC diagnosis.

Although not universally accepted, several authors have reported a higher sensitivity for dynamic contrastenhanced MRI compared with MDCT in the detection 
of hypervascular lesions. On the other hand, MRI offers advantages in terms of characterization of cell content and related to the development of new tools such as DWI and HB contrast agents. These new tools may be particularly helpful in the detection and characterization of borderline hypovascular lesions by providing functional information such as water molecule motion and cellular HB function, whose alterations can precede neoangiogenesis in the process of hepatocarcinogenesis (Table 1).

Also, newer CT technologies, represented by DECT, can increase hypervascular lesions' conspicuity, thus possibly improving CT sensitivity, particularly for small lesions. DECT might also provide newer quantitative parameters that reflect the degree of contrast uptake within a given volume and therefore can be representative of the arterial vascular supply.

More data and further developments are needed to verify the usefulness of these newer technologies in the diagnosis of $\mathrm{HCC}$ and to translate them into clinical practice.

Finally, there is an unmet need for standardization of liver imaging interpretation and reporting, particularly for atypical nodules, and scoring systems are now being developed and implemented in an attempt to reduce interobserver variability.

\section{Disclosure}

The authors report no conflicts of interest in this work.

\section{References}

1. Ferlay J, Shin HR, Bray F, Forman D, Mathers C, Parkin DM. Estimates of worldwide burden of cancer in 2008: GLOBOCAN 2008. Int J Cancer. 2010;127:2893-2917.

2. Llovet JM, Burroughs A, Bruix J. Hepatocellular carcinoma. Lancet. 2003;362(9399):1907-1917.

3. Sherman M. Hepatocellular carcinoma: epidemiology, surveillance, and diagnosis. Semin Liver Dis. 2010;30:3-16.

4. Fattovich G, Stroffolini T, Zagni I, et al. Hepatocellular carcinoma in cirrhosis: incidence and risk factors. Gastroenterology. 2004; 127(5 Suppl 1):S35-S50.

5. Davila JA, Morgan RO, Shaib Y, McGlynn KA, El-Serag HB. Hepatitis $C$ infection and the increasing incidence of hepatocellular carcinoma: a population-based study. Gastroenterology. 2004;127: 1372-1380.

6. Ascha MS, Hanouneh IA, Lopez R, Tamimi TA, Feldstein AF, Zein NN. The incidence and risk factors of hepatocellular carcinoma in patients with nonalcoholic steatohepatitis. Hepatology. 2010;51: 1972-1978.

7. Theise ND, Park YN, Kojiro M. Dysplastic nodules and hepatocarcinogenesis. Clin Liver Dis. 2002;6:497-512.

8. International Consensus Group for Hepatocellular Neoplasia. Pathologic diagnosis of early hepatocellular carcinoma: a report of the International Consensus Group for Hepatocellular Neoplasia. Hepatology. 2009;49: 658-664.

9. European Association for the Study of the Liver; European Organisation for Research and Treatment of Cancer. EASL-EORTC clinical practice guidelines: management of hepatocellular carcinoma. $J$ Hepatol. 2012;56:908-943.
10. Bruix J, Sherman M; American Association for the Study of Liver Diseases. Management of hepatocellular carcinoma: an update. Hepatology. 2011;53:1020-1022.

11. Omata M, Lesmana LA, Tateishi R, et al. Asian Pacific Association for the Study of the Liver consensus recommendations on hepatocellular carcinoma. Hepatol Int. 2010;4:439-474.

12. Terasaki S, Kaneko S, Kobayashi K, Nonomura A, Nakanuma Y. Histological features predicting malignant transformation of nonmalignant hepatocellular nodules: a prospective study. Gastroenterology. 1998;115:1216-1222.

13. Borzio M, Fargion S, Borzio F, et al. Impact of large regenerative, low grade and high grade dysplastic nodules in hepatocellular carcinoma development. J Hepatol. 2003;39:208-214.

14. Ricke J, Seidensticker M, Mohnike K. Noninvasive diagnosis of hepatocellular carcinoma in cirrhotic liver: current guidelines and future prospects for radiological imaging. Liver Cancer. 2012;1(1):51-58.

15. Bruix J, Sherman M, Llovet JM, et al. EASL Panel of Experts on HCC. Clinical management of hepatocellular carcinoma. Conclusions of the Barcelona-2000 EASL conference. European Association for the Study of the Liver. J Hepatol. 2001;35:421-430.

16. Yang ZF, Poon RT. Vascular changes in hepatocellular carcinoma. Anat Rec. 2008; 291(6):721-734.

17. Himeno H, Enzan H, Saibara T, Onishi S, Yamamoto Y. Hitherto unrecognized arterioles within hepatocellular carcinoma. J Pathol. 1994;174:217-222.

18. Matsui O. Analysis of intranodular hemodynamics of dysplastic nodules in cirrhotic livers. Radiology. 2001;218:603-605.

19. Ueda K, Terada T, Nakanuma Y, Matsui O. Vascular supply in adenomatous hyperplasia of the liver and hepatocellular carcinoma: a morphometric study. Hum Pathol. 1992;23:619-626.

20. Yamamoto T, Hirohashi K, Kaneda K, et al. Relationship of the microvascular type to the tumor size, arterialization and dedifferentiation of human hepatocellular carcinoma. Jpn J Cancer Res. 2001;92:1207-1213.

21. Kin M, Torimura T, Ueno T, Inuzuka S, Tanikawa K. Sinusoidal capillarization in small hepatocellular carcinoma. Pathol Int. 1994:44: 771-778.

22. Park YN, Yang CP, Fernandez GJ, Cubukcu O, Thung SN, Theise ND. Neoangiogenesis and sinusoidal "capillarization" in dysplastic nodules of the liver. Am J Surg Pathol. 1998;22:656-662.

23. Kudo M. Multistep human hepatocarcinogenesis: correlation of imaging with pathology. J Gastroenterol. 2009;44(Suppl 19):112-118.

24. Roskams T, Kojiro M. Pathology of early hepatocellular carcinoma: conventional and molecular diagnosis. Semin Liver Dis. 2010;30:17-25.

25. Matsui O, Kobayashi S, Sanada J, et al. Hepatocellular nodules in liver cirrhosis: hemodynamic evaluation (angiography-assisted CT) with special reference to multi-step hepatocarcinogenesis. Abdom Imaging. 2011;36:264-272.

26. Liu K, He X, Lei XZ, et al. Pathomorphological study on location and distribution of Kupffer cells in hepatocellular carcinoma. World $J$ Gastroenterol. 2003;9:1946-1949.

27. Park HS, Lee JM, Kim SH, et al. Differentiation of well-differentiated hepatocellular carcinomas from other hepatocellular nodules in cirrhotic liver: value of SPIO-enhanced MR imaging at 3.0 Tesla. J Magn Reson Imaging. 2009;29:328-335.

28. Bartolozzi C, Crocetti L, Lencioni R, Cioni D, Della Pina C, Campani D. Biliary and reticuloendothelial impairment in hepatocarcinogenesis: the diagnostic role of tissue-specific MR contrast media. Eur Radiol. 2007;17:2519-2530.

29. Forner A, Vilana R, Ayuso C, et al. Diagnosis of hepatic nodules $20 \mathrm{~mm}$ or smaller in cirrhosis: prospective validation of the noninvasive diagnostic criteria for hepatocellular carcinoma. Hepatology. 2008;47:97-104.

30. Sangiovanni A, Manini MA, Iavarone M, et al. The diagnostic and economic impact of contrast imaging techniques in the diagnosis of small hepatocellular carcinoma in cirrhosis. Gut. 2010;59(5): $638-644$. 
31. Kim K, Kim CS, Han YM, et al. Detection of hepatocellular carcinoma: gadoxetic acid-enhanced 3-dimensional magnetic resonance imaging versus multi-detector row computed tomography. J Comput Assist Tomogr. 2009;33:844-850.

32. Leoni S, Piscaglia F, Golfieri R, et al. The impact of vascular and nonvascular findings on the noninvasive diagnosis of small hepatocellular carcinoma based on the EASL and AASLD criteria. Am J Gastroenterol. 2010;105(3):599-609.

33. Bolondi L, Gaiani S, Celli N, et al. Characterization of small nodules in cirrhosis by assessment of vascularity: the problem of hypovascular hepatocellular carcinoma. Hepatology. 2005;42:27-34.

34. Roskams T. Anatomic pathology of hepatocellular carcinoma: impact on prognosis and response to therapy. Clin Liver Dis. 2011;15 245-259.

35. Park MJ, Kim YK, Lee MH, Lee JH. Validation of diagnostic criteria using gadoxetic acid-enhanced and diffusion-weighted MR imaging for small hepatocellular carcinoma $(\leq 2.0 \mathrm{~cm})$ in patients with hepatitisinduced liver cirrhosis. Acta Radiol. 2013;54(2):127-136.

36. Bota S, Piscaglia F, Marinelli S, Pecorelli A, Terzi E, Bolondi L. Comparison of international guidelines for noninvasive diagnosis of hepatocellular carcinoma. Liver Cancer. 2012;1:190-200.

37. Bruix J, Sherman M; Practice Guidelines Committee, American Association for the Study of Liver Diseases. Management of hepatocellular carcinoma. Hepatology. 2005;42(5):1208-1236.

38. Boozari B, Soudah B, Rifai K, et al. Grading of hypervascular hepatocellular carcinoma using late phase of contrast enhanced sonography: a prospective study. Dig Liver Dis. 2011;43:484-490.

39. Vilana R, Forner A, Bianchi L,et al. Intrahepatic peripheral cholangiocarcinoma in cirrhosis patients may display a vascular pattern similar to hepatocellular carcinoma on contrast-enhanced ultrasound. Hepatology. 2010;51:2020-2029.

40. Bolondi L, Cillo U, Colombo M, et al; Italian Association for the Study of the Liver (AISF); AISF Expert Panel; AISF Coordinating Committee. Position paper of the Italian Association for the Study of the Liver (AISF): the multidisciplinary clinical approach to hepatocellular carcinoma. Dig Liver Dis. 2013;45(9):712-723.

41. Barreiros AP, Piscaglia F, Dietrich CF. Contrast enhanced ultrasound for the diagnosis of hepatocellular carcinoma (HCC): comments on AASLD guidelines. J Hepatol. 2012;57:930-932.

42. Golfieri R, Renzulli M, Lucidi V, et al. Contribution of the hepatobiliary phase of Gd-EOB-DTPA-enhanced MRI to dynamic MRI in the detection of hypovascular small $(\leq 2 \mathrm{~cm}) \mathrm{HCC}$ in cirrhosis. Eur Radiol. 2011;21:1233-1242.

43. Rimola J, Forner A, Tremosini S, et al. Non-invasive diagnosis of hepatocellular carcinoma $\leq 2 \mathrm{~cm}$ in cirrhosis. Diagnostic accuracy assessing fat, capsule and signal intensity at dynamic MRI. J Hepatol. 2012;56:1317-1323.

44. Bartolozzi C, Battaglia V, Bargellini I, et al. Contrast-enhanced magnetic resonance imaging of 102 nodules in cirrhosis: correlation with histological findings on explanted livers. Abdom Imaging. 2013;38(2): 290-296.

45. Khalili K, Kim TK, Jang HJ, Yazdi LK, Guindi M, Sherman M. Indeterminate 1-2-cm nodules found on hepatocellular carcinoma surveillance: biopsy for all, some, or none? Hepatology. 2011;54: 2048-2054.

46. Ichikawa T, Haradome H, Hachiya J, et al. Diffusion weighted MR imaging with a single-shot echoplanar sequence: detection and characterization of focal hepatic lesions. Am J Roentgenol. 1998;170:397-402.

47. Gourtsoyianni S, Papanikolaou N, Yarmenitis S, et al. Respiratory gated diffusion-weighted imaging of the liver: value of apparent diffusion coefficient measurements in the differentiation between most commonly encountered benign and malignant focal liver lesions. Eur Radiol. 2008;18:486-492.

48. Geschwind JF, Artemov D, Abraham S, et al. Chemoembolization of liver tumor in a rabbit model: assessment of tumor cell death with diffusion-weighted MR imaging and histological analysis. J Vasc Inter Radiol. 2000;11:1245-1255.
49. Vandecaveye V, De Keyzer F, Verslype C, et al. Diffusion-weighted MRI provides additional value to conventional dynamic contrastenhanced MRI for detection of hepatocellular carcinoma. Eur Radiol. 2009;19:2456-2466.

50. Xu PJ, Yan FH, Wang JH, Shan Y, Ji Y, Chen CZ. Contribution of diffusion-weighted magnetic resonance imaging in the characterization of hepatocellular carcinomas and dysplastic nodules in cirrhotic liver. J Comput Assist Tomogr. 2010;34(4):506-512.

51. Wu LM, Xu JR, Lu Q, Hua J, Chen J, Hu J. A pooled analysis of diffusion-weighted imaging in the diagnosis of hepatocellular carcinoma in chronic liver diseases. J Gastroenterol Hepatol. 2013;28(2):227-234.

52. Kim YK, Lee WJ, Park MJ, Kim SH, Rhim H, Choi D. Hypovascular hypointense nodules on hepatobiliary phase gadoxetic acid-enhanced MR images in patients with cirrhosis: potential of DW imaging in predicting progression to hypervascular HCC. Radiology. 2012;265: 104-114.

53. Reimer P, Balzer T. Ferucarbotran (Resovist): a new clinically approved RES-specific contrast agent for contrast-enhanced MRI of the liver: properties, clinical development, and applications. Eur Radiol. 2003;13:1266-1276.

54. Lim JH, Choi D, Cho SK, et al. Conspicuity of hepatocellular nodular lesions in cirrhotic livers at ferumoxides-enhanced MR imaging: importance of Kupffer cell number. Radiology. 2001;220:669-676.

55. Chen RC, Lii JM, Chou CT, et al. T2-weighted and T1-weighted dynamic superparamagnetic iron oxide (ferucarbotran) enhanced MRI of hepatocellular carcinoma and hyperplastic nodules. J Formos Med Assoc. 2008;107(10):798-805.

56. Lutz AM, Willmann JK, Goepfert K, et al. Hepatocellular carcinoma in cirrhosis: enhancement patterns at dynamic gadolinium- and superparamagnetic iron oxide-enhanced T1-weighted MR imaging. Radiology. 2005;237:520-528.

57. Kirchin MA, Pirovano GP, Spinazzi A, et al. Gadobenate dimeglumine (Gd-BOPTA). An overview. Invest Radiol. 1998;33:798-809.

58. Kitao A, Zen Y, Matsui O, et al. Hepatocellular carcinoma: signal intensity at gadoxetic acid-enhanced MR imaging: correlation with molecular transporters and histopathologic features. Radiology. 2010;256:817-826.

59. Kitao A, Matsui O, Yoneda N, et al. The uptake transporter OATP8 expression decreases during multistep hepatocarcinogenesis: correlation with gadoxetic acid enhanced MR imaging. Eur Radiol. 2011;21:2056-2066.

60. Narita M, Hatano E, Arizono S, et al. Expression of OATP1B3 determines uptake of Gd-EOB-DTPA in hepatocellular carcinoma. J Gastroenterol. 2009;44:793-798.

61. Giovagnoni A, Paci E. Liver III. Gadolinium-based hepatobiliary contrast agents (Gd-EOB-DTPA and Gd-BOPTA/Dimeg). Magn Reson Imaging Clin N Am. 1996;4(1):61-72.

62. Ahn SS, Kim MJ, Lim JS, Hong HS, Chung YE, Choi JY. Added value of gadoxetic acid-enhanced hepatobiliary phase MR imaging in the diagnosis of hepatocellular carcinoma. Radiology. 2010;255:459-466.

63. Di Martino M, Marin D, Guerrisi A, et al. Intraindividual comparison of gadoxetate disodium-enhanced MR imaging and 64-section multidetector CT in the detection of hepatocellular carcinoma in patients with cirrhosis. Radiology. 2010;256:806-816.

64. Haradome H, Grazioli L, Tinti R, et al. Additional value of gadoxetic acidDTPA-enhanced hepatobiliary phase MR imaging in the diagnosis of earlystage hepatocellular carcinoma: comparison with dynamic triple-phase multidetector CT imaging. J Magn Reson Imaging. 2011;34:69-78.

65. Kim SH, Kim SH, Lee J, et al. Gadoxetic acid-enhanced MRI versus triple-phase MDCT for the preoperative detection of hepatocellular carcinoma. AJR Am J Roentgenol. 2009;192:1675-1681.

66. Di Martino M, De Filippis G, De Santis A, et al. Hepatocellular carcinoma in cirrhotic patients: prospective comparison of US, CT and MR imaging. Eur Radiol. 2013;23(4):887-896.

67. Inoue T, Kudo M, Komuta M, et al. Assessment of Gd-EOB-DTPAenhanced MRI for HCC and dysplastic nodules and comparison of detection sensitivity versus MDCT. J Gastroenterol. 2012;47:1036-1047. 
68. Kogita S, Imai Y, Okada M, et al. Gd-EOB-DTPA-enhanced magnetic resonance images of hepatocellular carcinoma: correlation with histological grading and portal blood flow. Eur Radiol. 2010;20:2405-2413.

69. Pastor CM. Gadoxetic acid-enhanced hepatobiliary phase MR imaging: cellular insight. Radiology. 2010;257:589.

70. Tsuboyama T, Onishi H, Kim T, et al. Hepatocellular carcinoma: hepatocyte-selective enhancement at gadoxetic acid-enhanced MR imaging-correlation with expression of sinusoidal and canalicular transporters and bile accumulation. Radiology. 2010;255:824-833.

71. Yoo SH, Choi JY, Jang JW, et al. Gd-EOB-DTPA-enhanced MRI is better than MDCT in decision making of curative treatment for hepatocellular carcinoma. Ann Surg Oncol. 2013;20:2893-2900.

72. Park MJ, Kim YK, Lee MW, et al. Small hepatocellular carcinomas: improved sensitivity by combining gadoxetic acid-enhanced and diffusion-weighted MR imaging patterns. Radiology. 2012;264: 761-770.

73. Flohr TG, Mccollough CH, Bruder H, et al. First performance evaluation of a dual-source CT (DSCT) system. Eur Radiol. 2006;16: 256-268.

74. Johnson TR, Krauss B, Sedlmair M, et al. Material differentiation by dual energy CT: initial experience. Eur Radiol. 2007;17:1510-1517.

75. Altenbernd J, Heusner TA, Ringelstein A, Ladd SC, Forsting M, Antoch G. Dual-energy-CT of hypervascular liver lesions in patients with HCC: investigation of image quality and sensitivity. Eur Radiol. 2011;21:738-743.

76. Lv P, Lin XZ, Chen K, Gao J. Spectral CT in patients with small HCC: investigation of image quality and diagnostic accuracy. Eur Radiol. 2012;22:2117-2124.

77. Lv P, Lin XZ, Li J, Li W, Chen K. Differentiation of small hepatic hemangioma from small hepatocellular carcinoma: recently introduced spectral CT method. Radiology. 2011;259:720-729.

78. Yu Y, He N, Sun K, Lin X, Yan F, Chen K. Differentiating hepatocellular carcinoma from angiomyolipoma of the liver with CT spectral imaging: a preliminary study. Clin Radiol. 2013;68:e491-e497.
79. Yu Y, Lin X, Chen K, et al. Hepatocellular carcinoma and focal nodular hyperplasia of the liver: differentiation with CT spectral imaging. Eur Radiol. 2013;23:1660-1668.

80. Dai X, Schlemmer HP, Schmidt B, et al. Quantitative therapy response assessment by volumetric iodine-uptake measurement: initial experience in patients with advanced hepatocellular carcinoma treated with sorafenib. Eur J Radiol. 2013;82:327-334.

81. Organ Procurement and Transplantation Network. Policy 09: Allocation of Livers and Liver-Intestines. Available from: http:// optn.transplant.hrsa.gov/ContentDocuments/OPTN_Policies. pdf\#nameddest=Policy_09. Accessed August 5, 2014.

82. Davenport MS, Khalatbari S, Liu PS, et al. Repeatability of diagnostic features and scoring systems for hepatocellular carcinoma by using MR imaging. Radiology. Epub February 18, 2014.

83. Fowler KJ, Karimova EJ, Arauz AR, et al. Validation of Organ Procurement and Transplant Network (OPTN)/United Network for Organ Sharing (UNOS) criteria for imaging diagnosis of hepatocellular carcinoma. Transplantation. 2013;95(12):1506-1511.

84. Purysko AS, Remer EM, Coppa CP, Leão Filho HM, Thupili CR, Veniero JC. LI-RADS: a case-based review of the new categorization of liver findings in patients with end-stage liver disease. Radiographics. 2012;32(7):1977-1995.

85. American College of Radiology. Liver Imaging Reporting and Data System Version 2013.1. Available from: http:/www.acr.org/QualitySafety/Resources/LIRADS/. Accessed August 5, 2014.

86. Tang A, Cruite I, Sirlin CB. Toward a standardized system for hepatocellular carcinoma diagnosis using computed tomography and MRI. Expert Rev Gastroenterol Hepatol. 2013;7(3):269-279.

87. Clark TJ, McNeeley MF, Maki JH. Design and implementation of handheld and desktop software for the structured reporting of hepatic masses using the LI-RADS schema. Acad Radiol. 2014;21(4): 491-506.
Journal of Hepatocellular Carcinoma

\section{Publish your work in this journal}

The Journal of Hepatocellular Carcinoma is an international, peerreviewed, open access journal that offers a platform for the dissemination and study of clinical, translational and basic research findings in this rapidly developing field. Development in areas including, but not limited to, epidemiology, vaccination, hepatitis therapy, pathology and

\section{Dovepress}

molecular tumor classification and prognostication are all considered for publication. The manuscript management system is completely online and includes a very quick and fair peer-review system, which is all easy to use. Visit http://www.dovepress.com/testimonialsphp to read real quotes from published authors. 